\title{
Many-objective optimization of build part orientation in additive manufacturing
}

\author{
Marina A. Matos • Ana Maria A. C. Rocha .
}

Lino A. Costa

the date of receipt and acceptance should be inserted later

Abstract Additive manufacturing is the process of building a three-dimensional object from a computer-aided design model, by successively adding material layerby-layer. This technology allows to print complex shape objects and is being rapidly adopted throughout the aircraft industry, medical implants, jewelry, footwear industry, automotive industry and fashion products.

The build orientation of 3D objects has a strong influence on many quality characteristics. In this paper, a many-objective approach is applied to the Fin model, using the NSGA-II algorithm to optimize four conflicting objective functions regarding the need for support structures, the build time, the surface roughness and the overall quality of the surface.

First, a bi-objective optimization is performed for each couple of two objectives and some representative solutions are identified. However, when applying manyobjective optimization to the four objective functions simultaneously, some more orientation angles are found as good optimal solutions. Visualization tools are used to inspect the relationships and the trade-offs between the objectives. Then, the decision-maker can choose which orientation angles are more favorable according to his/her preferences. The optimal solutions found confirmed the effectiveness of the proposed approach.

Keywords Additive manufacturing - 3D printing - Multi-objective Optimization . Build orientation

Marina A. Matos

ALGORITMI Center, University of Minho, 4710-057 Braga, Portugal

E-mail: aniram@live.com.pt

Ana Maria A. C. Rocha

ALGORITMI Center, University of Minho, 4710-057 Braga, Portugal

E-mail: arocha@dps.uminho.pt

Lino A. Costa

ALGORITMI Center, University of Minho, 4710-057 Braga, Portugal

E-mail: lac@dps.uminho.pt 


\section{Introduction}

Additive manufacturing ( $\mathrm{AM}$ ) is a process that builds 3D objects, from 3D model data, by adding layer-by-layer of material. AM uses a wide variety of construction materials, such as plastic, resin, rubber, ceramics, glass, concrete and metal. One of the greatest benefits of AM is the production of a wide range of shapes. This process begins with a 3D mesh model that can be created by structures built in computer-aided design (CAD) software and then creates an STL (Surface Tessellation Language) file.

The reduction of the development time of the prototype model through the adoption of techniques to manufacture a prototype model from a CAD file, using the additive manufacturing process, is one of the current challenges faced by the manufacturing industries. The scanning technology of an object, CAD model, aims to improve the quality of the model allowing to view and operate the object in real time (Chong et al., 2018). Recently, AM is being used to fabricate end-use products in several areas, such as aircraft, dental restorations, medical implants, automobiles and fashion products (Khan et al., 2017).

The surface finish of additive manufactured parts can be affected by different factors, from pre-processing, processing and post-processing steps. The poor surface finish in AM processes can be affected by the tessellation of the original CAD model and the slicing procedure used during the building process. Although a reduction in the layer thickness may cause an improvement in the surface roughness it increases the build time. Post-processing surface treatment results in additional build time and costs, and leads to a degradation of the geometrical definition of the model (Vahabli \& Rahmati, 2017).

Some authors have demonstrated that an optimized building direction can improve the surface finish of an object (Ahn et al., 2008, Behnam, 2011, Byun \& Lee, 2006, Campbell et al., 2002, Li et al., 2010; Pandey et al., 2003, Vahabli \& Rahmati, 2017, Wang et al., 2016). The build orientation of a given part is a very important factor that influences different parameters, such as the staircase effect, the support volume, the support area, the number of supports required, the build time, the surface roughness, the surface quality, process planning, post-processing and cost (Y. Zhang, De Backer, et al., 2016). Several works were presented by different authors with the objective of obtaining the optimal build orientation of a 3D model in order to improve the printing process of this model (Taufik \& Jain, 2013, Xu et al., 1997, Jibin, 2005).

The work presented by Sumalatha and Rao (2018) aims to find an optimal model orientation in order to minimize the amount of support material needed for the printing of the object. A smaller amount of support material means a reduction in post-processing costs and an increase in accuracy and surface finish. The optimal orientation in order to minimize the support volume of $3 \mathrm{D}$ models was studied by Mirzendehdel and Suresh (2016). Pereira et al. (2018) optimized the object building orientation and the need for supports generation. They found that the smaller the number of supports the better the accuracy and smoothness of the surface of the object. Two global optimization methods, Electromagnetism and Stretch Simulated Annealing algorithms, were used to find the best build orientation of four models, considering the staircase effect, support area and build time can be found in the study (Rocha et al., 2018). They concluded that both methods have the ability to solve the problem and were able to find several optimal solu- 
tions. Matos, Rocha, and Pereira (2019) performed a single-objective work in order to obtain the optimal build orientation, using the Genetic Algorithm (GA). They considered three objective functions, the volumetric error, the support area and the build time. Several optimal solutions were obtained using GA. An optimization model developed to improve the build orientation based on the minimization of support structures can be found in (Das et al., 2015).

In a single-objective approach, we may achieve a build orientation that satisfies one or two criteria but does not meet them simultaneously. For instance, a good orientation angle providing the construction of the object in a fast time could lead to a big structure of supports affecting the surface finish of the object. Thus, multi-objective approaches to determine the optimal object building orientation in the construction of CAD models have been developed (Canellidis et al. 2006, Ransikarbum et al., 2017, Thrimurthulu et al., 2004). In this way, the build orientation problem can be divided into two essential tasks. The first is based on the identification of a set of alternative build orientations for a $3 \mathrm{D}$ object. The second task is the application of a multi-objective decision-making method, with the ability to determine the best alternatives considering different options (Byun \& Lee, 2006, Cheng et al., 1995, Y. Zhang \& Bernard, 2013, Y. Zhang, Bernard, et al., 2016).

Vahabli and Rahmati (2017) proposed a hybrid approach to obtain surface roughness values for different build angles. In this experimental research they found that the larger the build angle the greater the surface roughness. In addition, they concluded that reducing the layer thickness increases the build time but improves the surface roughness of the model. An aggregated artificial neural network to investigate the simultaneous effects of layer thickness and print orientation of porous structures was developed by Asadi-Eydivand et al. (2016), thus verifying the compressive strength and porosity of the layers. The particle swarm optimization algorithm was used to approximate the Pareto front. They concluded that decreasing the orientation angles also decreases the delay time in moving to a new layer and also decreases the compression force. In contrast, the porosity of the object increases.

Golmohammadi and Khodaygan (2019) minimized a bi-objective optimization problem based on the amount of supports and the surface roughness. The obtained results showed that the proposed method produced a better part build orientation compared to the conventional methods. A multi-objective problem was also presented by X. Zhang et al. (2015), where the objectives were to minimize the support area, the visual salience, the preferred point of view and the preservation of smoothness. They concluded that the applied model was a success because it was able to minimize the visual impact of the part. Brika et al. (2017) used a genetic algorithm and considered mechanical properties, support structures, surface roughness, build time and cost in order to optimize the build orientation. In (Phatak \& Pande, 2012) a genetic algorithm was used to optimize a weighted average of five normalized evaluation criteria (build height, staircase error, material utilization, surface area in contact with support structures and volume of support structures) based on their relevance to the rapid prototyping process. They concluded that the optimization of the considered parameters provided significant improvements in part productivity, quality and economy during part fabrication.

Gurrala and Regalla (2014) applied the NSGA-II algorithm to optimize the strength of the model and its volumetric shrinkage as objective functions. Through 
the Pareto front, they concluded that with the shrinkage of the part its strength increases in the horizontal and vertical directions. Ga et al. (2019) proposed a multi-objective strategy using a weighted sum method in order to reduce the support volume, build time and costs and increase the surface quality, depending on the orientation chosen by the user and the weights associated with each objective function. Matos, Rocha, Costa, and Pereira (2019) proposed a multi-objective problem using the Tchebycheff method with the Electromagnetism-like algorithm to minimize the area of the object in contact with the supporting structures and the build time of four 3D models. The study involved the combination between two objectives. They analyzed the results through Pareto fronts, concluding that it is possible to identify the trade-offs between the objectives and select the most appropriate solution.

The main goal of this paper is to optimize the part build orientation considering four criteria in order to provide the decision-maker the optimal build orientations and their trade-offs in printing the 3D Fin model. As far as we know, the build orientation problem has not yet been addressed as a many-objective optimization problem. In this paper, the many-objective approach involves four objective functions to be simultaneously optimized: support area (SA), build time (BT), surface roughness (RA) and surface quality (SQ).

This paper is organized as follows. Section 2 introduces the effects of the build orientation and the 3D model to be tested. In Sect. 3, the many-objective approach is presented comprising the description of the objective functions as well as algorithm used in the study. The results obtained considering different bi-objective problems and the many-objective problem are presented in Sect. 4 . Finally, Sect. 5 concludes the paper.

\section{Build orientation}

In this section, the staircase effect associated with the build orientation and the 3D Fin model are briefly presented.

\subsection{Staircase effect}

The object accuracy in AM is highly dependent on its build orientation. The quality of an object produced can be influenced by many different parameters, in particular the layer thickness. The number of layers required to construct an object affects the printing speed and the final quality of the object. The impact of layer thickness is more noticeable on curves and angles and is less prominent on straight vertical walls, due to the layer-by-layer nature of $3 \mathrm{D}$ printing.

The staircase effect occurs because different layers can stay inside or outside the contours of the original models, giving the perception of a layer, as can be seen in Fig. 1 (represented in two dimensions for a better visualization), where the original model is represented by a semi-circle and the layers are represented by rectangles. This effect results from the representation of curved objects by layers. This effect is verified when the layer marks between the model surface and the part surface become distinctly visible on the surface of the parts. The error associated with the staircase effect is due to the layer thickness and the slope of 
the part surface (Jaiswal et al., 2018). The maximum deviation between the model surface and the printed object, caused by the staircase effect, is calculated by the maximum deviation from the layered part to the CAD surface measured in the normal direction to CAD surface.

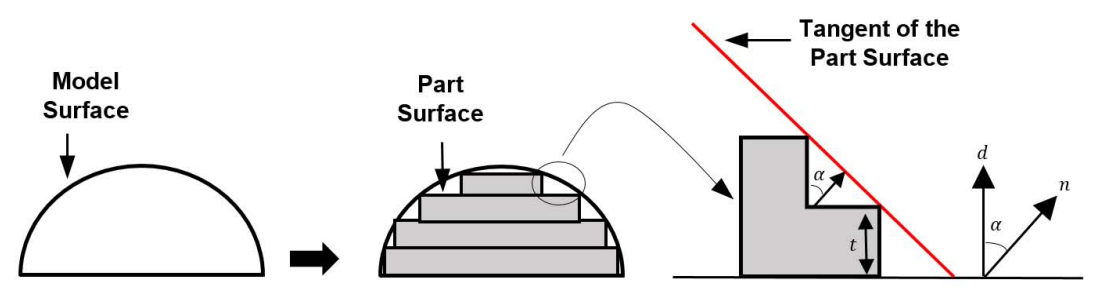

Fig. 1 Staircase effect scheme

The angle $\alpha$ is formed by the slicing direction $d$ and the model surface normal $n$, and on the layer thickness $t$. Thicker layers and/or higher values of $\cos (\alpha)$ will produce larger values for cusp height $(t \cos (\alpha))$ and consequently a more inaccurate surface appears (Alexander et al., 1998, Thrimurthulu et al., 2004, Pereira et al. 2018).

\subsection{Fin model}

The 3D CAD model that will be used in this study is the Fin model.

Additive manufacturing uses 3D CAD files converted in the form of a STL (Standard Tessellation Language) file, since it is the industry standard file type for 3D Printing. The STL file format uses a polyhedral representation of a 3D object based on triangular facets to represent the surfaces of a solid model. To approximate the surface of a solid model, triangular facets are used for the polyhedral representation of a 3D object and the coordinates of the vertices in text format are defined in the STL file. The STL files describe only the surface geometry, not representing color, texture, or other common attributes of the CAD model. The more complex the model is, the greater the number of triangular facets.

Figure 2(a) depicts the Fin model, in the STL file format. As can be seen, the model is symmetrical, so different orientations of the model on the $x$-axis and $y$-axis (keeping the $z$-axis fixed) can give the same build orientation.

The size of the Fin model is $121.5 \times 53.9 \times 16$ as width $\times$ height $\times$ depth measures in $\mathrm{mm}$ and the volume is $33.3 \mathrm{~cm}^{3}$. The number of triangles is 15370 and the number of slices is 256 for a layer thickness of $0.2 \mathrm{~mm}$ (layer thickness used in this work), as can be seen in Fig. 2(b).

\section{Many-objective approach}

This section begins by formulating the many-objective optimization problem followed by the description of the four quality measures, or objective functions, used in the build orientation problem. Then, the multi-objective genetic algorithm used to solve the optimization problem is briefly described. 


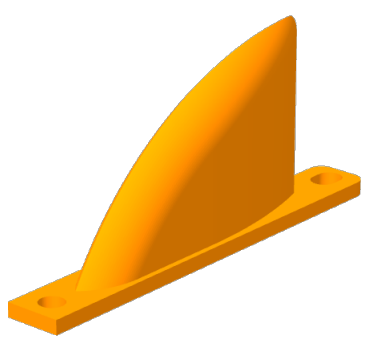

(a) Fin model

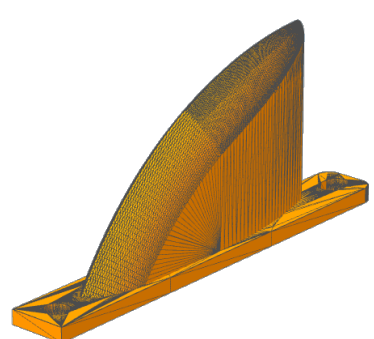

(b) Fin in triangles format

Fig. 2 Fin model used in the study

\subsection{Problem formulation}

The optimization problem aiming to solve the build orientation problem of a 3D CAD model is related to the computation of the optimal slicing direction $d$, which is a normalized vector (i.e. $\|d\|=1$ ). In order to define the build orientation problem of a model, it is only required to consider the rotation angle $\theta_{x}$ of the model around $x$-axis and $\theta_{y}$ around $y$-axis, because in 3D printed layer manufacturing the rotation of a model around the $z$-axis does not affect the the construction process. In this study, the direction $d=(0,0,1)^{T}$ was considered as the slicing direction after a rotation along $\theta=\left(\theta_{x}, \theta_{y}\right)$ angles, where each angle is between $0^{\circ}$ and $180^{\circ}$.

The mathematical formulation of the optimization problem is given by

$$
\begin{array}{ll}
\min & \left\{f_{1}\left(\theta_{x}, \theta_{y}\right), \ldots, f_{k}\left(\theta_{x}, \theta_{y}\right)\right\} \\
\text { s.t. } & 0 \leq \theta_{x} \leq 180 \\
& 0 \leq \theta_{y} \leq 180
\end{array}
$$

where $k$ is the number of objective functions and $\theta_{x}$ and $\theta_{y}$ are the rotation angles along the $x$-axis and the $y$-axis, respectively.

The object orientation in the AM process is one of the key elements, which can have a significant effect on the quality of produced object, support requirement, build time, part stability, etc. The objective functions used in this study are: the support area, the build time, the surface roughness and the surface quality.

\subsubsection{Support area}

The part orientation plays an important role in defining the amount of supports required to sustain overhanging parts. When adding a support structure to a part, the print time will be longer since the support structure also needs to be printed. In addition, the support structures can affect the surface finish when the supports are removed.

The amount of supports can be measured by the support area or support volume. The support volume is the volume of the region that is between the layer under construction and the platform of the $3 \mathrm{D}$ printer, and is computationally very complex to calculate. The support area mainly affects post-processing and surface finish and can be measured through the total contact area of the external 
supports with the object. Computationally, the support area is simpler and more important than the support volume when it comes to part accuracy (Rocha et al. 2018, Jibin, 2005).

The support area, can be mathematically formulated as

$$
S A=\sum_{i} A_{i}\left|d^{T} n_{i}\right| \delta
$$

where $A_{i}$ is the area of each triangular facet $i, n_{i}$ is the normal unit vector of each triangular facet $i, d$ is the unit vector of the direction of construction and $\delta=1$ if $d^{T} n_{i}<0$ otherwise $\delta=0$ (Jibin, 2005).

Figure 3 shows the $S A$ objective function landscape for the Fin model. It can be observed that the $S A$ function is nonconvex with multiple local optima.

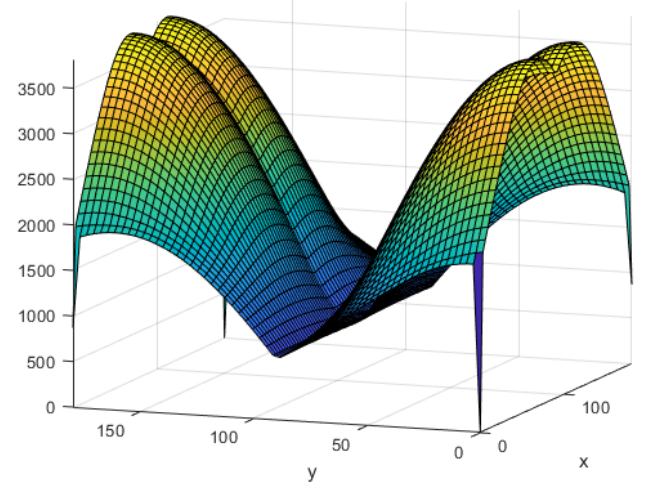

Fig. $3 S A$ objective function

In order to know the optimal solution for each objective function, the ga function from MATLAB Global Optimization Toolbox ${ }^{\circledR}($ MATLAB, 2019) that implements the Genetic Algorithm is used. GA is a stochastic, population-based algorithm based on the natural selection process that mimics biological evolution. GA randomly selects individuals from a current population and uses them as parents to produce the children for the next generations. Over successive generations, the population "evolves" towards an optimal solution randomly searching by mutation and crossover among population members (Goldberg, 1989).

In this work, a population size of 50 individuals and the maximum number of generations of 200 as stopping criterion is considered, when running GA. The optimal solution obtained for $S A$ function is $\left(\theta_{x}, \theta_{y}\right)=(0.00,0.00)$, that is equivalent to the solution $\left(\theta_{x}, \theta_{y}\right)=(180.00,180.00)$.

\subsubsection{Build time}

The time required to create an object accurately is composed of the time of the $3 \mathrm{D}$ printer to deposit the material layer-by-layer plus the time for support removal and surface finishing. The largest slice of time spent to build the object is due to 
the deposition time, while the time to remove support structures and finish the surface is only a smaller fraction of the overall time Canellidis et al. (2006).

In Jibin (2005), the build time encompasses the precise time for the platform to move downwards during the construction of each layer, which depends on the total number of slices of the solid. On the other hand, the number of slices depends on the height of the construction orientation of the object, thus the build time is proportional to the height of the model. Therefore, minimizing the height and the number of layers, can decrease the build time of the solid.

The build time, is given by

$$
B T=\max _{i}\left(d^{T} v_{i}^{1}, d^{T} v_{i}^{2}, d^{T} v_{i}^{3}\right)-\min _{i}\left(d^{T} v_{i}^{1}, d^{T} v_{i}^{2}, d^{T} v_{i}^{3}\right)
$$

where $d$ is the direction vector and $v_{i}^{1}, v_{i}^{2}, v_{i}^{3}$ are the vertex triangle facets $i$.

Figure 4 shows the $B T$ objective function, which is nonconvex with multiple local optima. The angle $\left(\theta_{x}, \theta_{y}\right)=(90.00,180.00)$ (equivalent to $\left(\theta_{x}, \theta_{y}\right)=$

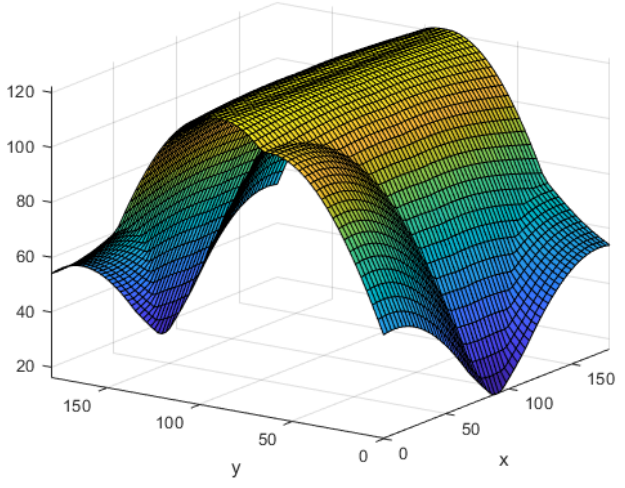

Fig. $4 B T$ objective function

$(90.00,0.00))$ is the optimal solution in terms of $B T$. This solution was obtained with GA using the same parameters applied for $S A$ function.

\subsubsection{Surface roughness}

The surface roughness can be affected by factors such as the layer thickness and the support structures (Jaiswal et al., 2018; Kattethota \& Henderson, 1998; Y. Zhang et al. 2017) and consequently by the part orientation.

Several authors have presented different approaches to define the surface roughness (Ahn et al., 2008; Byun \& Lee, 2006, Campbell et al., 2002, Canellidis et al. 2009 Mason, 2007). Mohan Pandey et al. (2003) and Pandey et al. (2003) formalized one of the most accepted strategies for the computation of surface roughness, given a certain range of angles. In (Behnam, 2011), a review on surface roughness formulas and a combination of strategies to evaluate the surface roughness value are proposed. 
The surface roughness for each triangle facet $i, R A_{i}$, is defined taking into consideration the build angle $\theta$, and is given by

$$
R A_{i}= \begin{cases}70.82 \frac{t}{\cos \left(\theta_{i}\right)}, & \text { if } 0^{\circ} \leq \theta_{i} \leq 70^{\circ} \\ \frac{1}{20}\left(90 R A_{i}^{70}-70 R A_{i}^{90}+\theta_{i}\left(R A_{i}^{90}-R A_{i}^{70}\right)\right) & \text { if } 70^{\circ}<\theta_{i}<90^{\circ} \\ 117.6 t, & \text { if } \theta_{i}=90^{\circ} \\ R A_{i}^{\theta_{i}-90}(1+w), & \text { if } 90^{\circ}<\theta_{i} \leq 135^{\circ} \\ \frac{1000}{2} t\left|\frac{\cos \left(\left(90-\theta_{i}\right)-\phi\right)}{\cos (\phi)}\right|, & \text { if } 135^{\circ}<\theta_{i} \leq 180^{\circ}\end{cases}
$$

where $t$ is the thickness of the layer, $\theta_{i}=90-\alpha_{i}$, where $\alpha_{i}$ is the angle of the unit vector of the direction and the normal unit vector for each triangle facet $i, R A_{i}^{70}$ and $R A_{i}^{90}$ are the values of $R A_{i}$ when $\theta_{i}=70^{\circ}$ and $\theta_{i}=90^{\circ}$, respectively. The $w$ is a dimensionless adjustment parameter for supported facets, $\phi$ is a phase shift in the range of $5^{\circ} \leq \phi \leq 15^{\circ}$ depending on the layer thickness (Behnam, 2011). The value 70.82 in the first branch of (4) refers to a value inside the confidence interval (69.28 72.36) used in Pandey (2010), $w=0.2$ as proposed in Pandey et al. (2003) and $\phi=5^{\circ}$ as in Behnam (2011).

The average surface roughness, considering the area of the triangular facets, can be calculated by

$$
R A=\frac{\sum_{i}\left(R A_{i} A_{i}\right)}{\sum_{i} A_{i}}
$$

where $R A_{i}$ is the roughness (in $\mu m$ ) of each triangular surface $i$ and $A_{i}$ is the area of triangular facet $i$. The smaller the $R A$ values, the smoother the surface is.

Figure 5 depicts the behavior of the $R A$ function and shows that is a nonconvex function with multiple local optima. The solution angle $\left(\theta_{x}, \theta_{y}\right)=(0.02,72.59)$

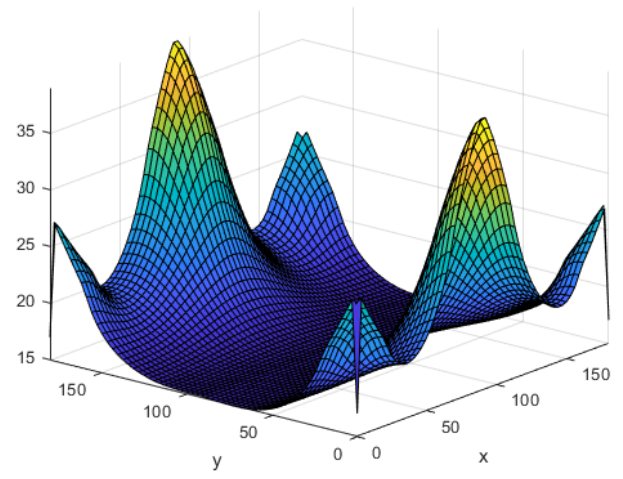

Fig. $5 R A$ objective function

was obtained with GA using the same parameters applied for $S A$ function. This solution is equivalent to $\left(\theta_{x}, \theta_{y}\right)=(179.98,107.41)$. 


\subsubsection{Surface quality}

The overall surface quality is one of the most studied characteristics in 3D printing processes that can be affected by the part orientation (Frank \& Fadel, 1995. Alexander et al., 1998, Wang et al., 2016, Xu et al., 1997). Several studies focused their work on the surface finish optimizing the building time, accuracy and stability of the part (Masood et al., 2000, Thrimurthulu et al., 2004).

One of the main issues affecting the surface finish is the staircase effect (see Fig. 1), that is influenced by the thickness of the layers. The smaller the layer thickness, the smaller staircase effect, leading to a better surface finish. This effect is related to the cusp height that is the maximum distance between the part surface and the model surface (Livesu et al. 2017). By using the cusp height, the surface quality can be determined from the object geometry, build direction and layer thickness (Matos et al., 2020).

The global surface quality measure, is defined by integrating the cusp height over the entire surface and given by

$$
S Q=\frac{\sum_{i}\left|\cos \left(\alpha_{i}\right)\right| t A_{i}}{\sum_{i} A_{i}}
$$

where $t$ is the layer thickness, $\alpha_{i}$ is the angle between the normal of the surface and the printing direction and $A_{i}$ is the area for each triangle $i$.

In Figure 6 it is observed that the $S Q$ function is a nonconvex function with multiple local optima. The optimal $S Q$ solution is given by $\left(\theta_{x}, \theta_{y}\right)=\left(\theta_{x}, 90\right)$,

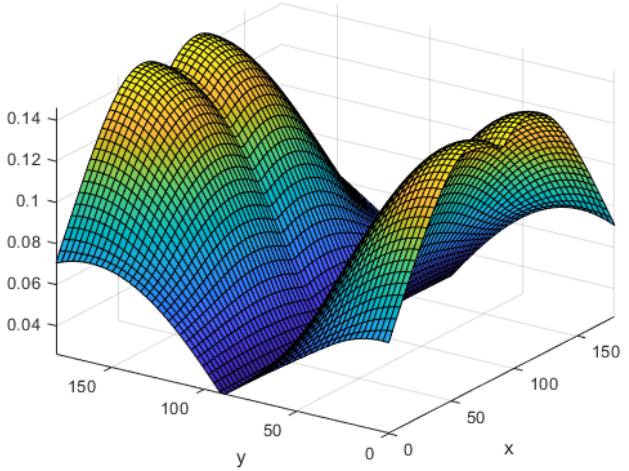

Fig. $6 S Q$ objective function

representing all solutions for which $\theta_{x} \in[0,180]$ and $\theta_{y}=90$, being obtained by the GA with the same parameters used in the optimization of the previous objective functions. 


\subsection{Multi-objective Genetic Algorithm}

In this work, the elitist Non-dominated Sorting Genetic Algorithm (NSGA-II) proposed by Deb (2001) is used. This is a multi-objective genetic algorithm that mimics the natural evolution of the species. Evolution starts from a population of individuals randomly generated, where each individual represents a potential solution of the multi-objective optimization problem. In NSGA-II, each individual in the current population is evaluated using a Pareto ranking and a crowding measure. First the best rank is assigned to all the non-dominated individuals in the current population. Solutions with the best rank are removed from the current population. Next, the second best rank is assigned to all the non-dominated solutions in the remaining population. In this manner, ranks are assigned to all solutions in the current population. The fittest individuals have a higher probability of being selected to generate new ones by genetic operators. NSGA-II uses a binary tournament selection based on non-domination rank and crowding distance to select a set of parent solutions. When two solutions are selected, the one with the lowest non-domination rank is preferred. Otherwise, if both solutions belong to the same rank, then the solution with the higher crowding distance is selected. Next, genetic operators such as recombination and mutation are applied to create an offspring population. Then, the two populations are merged together to form a combined population that is sorted according to different non-dominated fronts. If the size of the first non-dominated front is smaller then the population size, all members of this front are chosen for the new population. The remaining members of the population are chosen from subsequent non-dominated fronts in the order of their ranking.

The MATLAB ${ }^{\circledR}$ function gamultiobj provided in the Global Optimization Toolbox (MATLAB 2019) will be used in order to approximate the Pareto fronts. The gamultiobj function implements a multi-objective genetic algorithm that is a variant of the elitist NSGA-II (Deb, 2001). This function provides a set of algorithm options related with customizing randomization key properties, algorithm properties and termination criteria.

\section{Optimization results}

In this work, the goal is to find different trade-offs between the quality measures: support area, build time, surface roughness and surface quality for the Fin model. Therefore, there are conflicting objectives and different trade-off solutions that represent different compromises between the objectives. In this section, the optimization problem in (1) is firstly solved for all possible combinations of two objective functions, resulting in six different bi-objective optimization problems. Then, the four objectives $(S A, B T, R A$ and $S Q$ ) are optimized simultaneously and the results of the many-objective problem are presented, discussed and compared with those obtained in the bi-objective problems. 
4.1 Bi-objective results

Six bi-objective optimization problems were formulated for all possible pairs combinations of the four objectives (the support area, the build time, the surface roughness and the surface quality):

- $S A$ vs $B T$ - problem (1) with $f_{1}=S A$ and $f_{2}=B T$;

- $S A$ vs $R A$ - problem (1) with $f_{1}=S A$ and $f_{2}=R A$;

- $S A$ vs $S Q$ - problem (1) with $f_{1}=S A$ and $f_{2}=S Q$;

- $B T$ vs $R A$ - problem (1) with $f_{1}=B T$ and $f_{2}=R A$;

- $B T$ vs $S Q$ - problem (1) with $f_{1}=B T$ and $f_{2}=S Q$

- RA vs $S Q$ - problem (1) with $f_{1}=R A$ and $f_{2}=S Q$.

The MATLAB ${ }^{\circledR}$ gamultiobj function was used to solve the bi-objective optimization problems. The population size and the maximum number of generations was set to 50 (default value) and 600, respectively. Due to the stochastic nature of the optimization algorithm, 30 independent runs were performed. By default, the Pareto fraction is 0.35 and therefore, in each run, 18 non-dominated solutions are found $(0.35 \times$ population size). The Simplify $3 D$ software (a $3 \mathrm{D}$ model printing simulator) SIMPLIFY3D (2017) allows to visualize the solutions found for the Fin model.

Thereafter, the results obtained for the $S A$ vs $B T, S A$ vs $R A, S A$ vs $S Q, B T$ vs $R A, B T$ vs $S Q$, and $R A$ vs $S Q$ problems are presented and discussed. In all graphs, the set of non-dominated solutions obtained among the 30 independent runs are plotted with a blue dot. It should be noted that most of these solutions are not optimal and do not belong to the Pareto front. Thus, from this overall set of solutions, the non-dominated ones were determined and marked with a red circle defining the Pareto front. Representative solutions are selected to discuss trade-offs between objectives and identify the characteristics associated with these solutions.

\subsection{1 $S A$ vs $B T$}

Figure 7 shows the Pareto front obtained for $S A$ vs $B T$ problem. Representative non-dominated solutions $\mathrm{A}$ and $\mathrm{H}$ were selected. Dominated solutions $\mathrm{C}, \mathrm{E}$, and $\mathrm{K}$ are also marked since they correspond to non-dominated solutions when all objectives are optimized simultaneously (see Table 6). Table 1 shows the orientation angles and objectives values for the representative solutions.

Table 1 Representative dominated and non-dominated solutions for the $S A$ vs $B T$ problem

\begin{tabular}{crrrr}
\hline Solutions & \multicolumn{1}{c}{$\theta_{x}$} & \multicolumn{1}{c}{$\theta_{y}$} & \multicolumn{1}{c}{$S A$} & \multicolumn{1}{c}{$B T$} \\
\hline $\mathrm{A}$ & 0.00 & 0.02 & 0.0940 & 53.9497 \\
$\mathrm{C}$ & 94.80 & 90.00 & 594.2050 & 121.5000 \\
$\mathrm{E}$ & 0.00 & 7.58 & 1841.1001 & 66.3335 \\
$\mathrm{H}$ & 90.00 & 180.00 & 2637.4021 & 15.9888 \\
$\mathrm{~K}$ & 89.90 & 179.93 & 3542.0142 & 16.1397 \\
\hline
\end{tabular}

It can be observed that $S A$ increases from solution A to K (see also Table 1). Solutions C, E and $\mathrm{K}$ are dominated solutions when just these two objectives are 


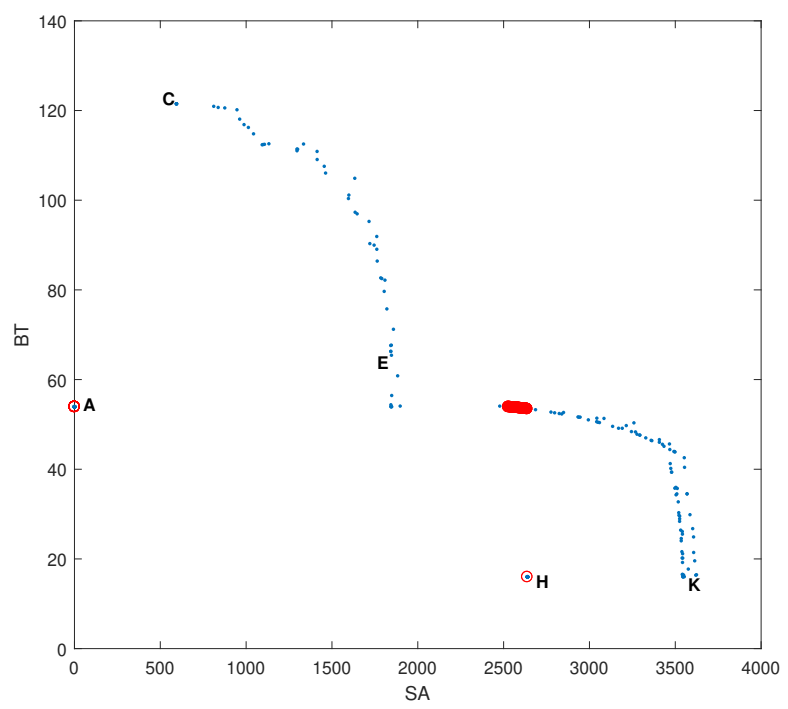

Fig. 7 Pareto front for the $S A$ vs $B T$ problem

considered. Solution A dominates solutions $\mathrm{C}$ and E since it is better in both objectives. Solution $\mathrm{K}$ is dominated by solution $\mathrm{H}$ since it is worse in both objectives.

In terms of orientation angles of the part, the part starts with an orientation of $(0.00,0.02)$ and, gradually, is rotated until being totally lied down as it can be observed in Fig. 13(a) and Fig. 13(g).

\subsection{2 $S A$ vs $R A$}

Figure 8 depicts the Pareto front obtained for the problem $S A$ vs $R A$ and Table 2 shows the orientation angles and objective values of the solutions pointed out in the graph.

Table 2 Representative non-dominated solutions for the $S A$ vs $R A$ problem

\begin{tabular}{crrrr}
\hline Solutions & \multicolumn{1}{c}{$\theta_{x}$} & \multicolumn{1}{c}{$\theta_{y}$} & \multicolumn{1}{c}{$S A$} & \multicolumn{1}{c}{$R A$} \\
\hline $\mathrm{A}$ & 0.00 & 0.02 & 0.0940 & 27.9241 \\
$\mathrm{~B}$ & 0.00 & 0.00 & 0.1313 & 17.0037 \\
$\mathrm{C}$ & 94.80 & 90.00 & 594.2050 & 15.1459 \\
$\mathrm{D}$ & 179.99 & 107.41 & 966.7508 & 14.9626 \\
\hline
\end{tabular}

All solutions A to D are non-dominated solutions in terms of these objectives. Solution A and D are the extremes of the Pareto front. Solutions A and B have similar orientations and close values of $S A$ with very different values of $R A$. Solution $\mathrm{C}$ is slightly worse than solution $\mathrm{B}$ in terms of $R A$ but considerably better in terms of $S A$. These solutions are shown in Fig. 13(a), 13(b) and Fig. 13(c). 


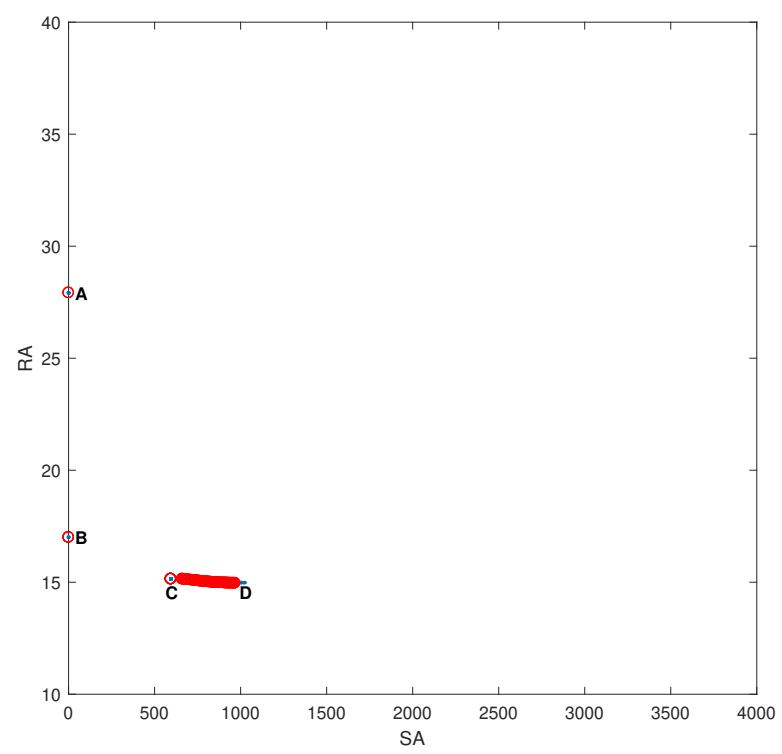

Fig. 8 Pareto front for the $S A$ vs $R A$ problem

\subsection{3 $S A$ vs $S Q$}

For the problem $S A$ vs $S Q$, just two non-dominated solutions were found: $A=$ $(0.00,0.02)$ e $C=\left(\forall \theta_{x}, 90\right)$. The first solution has fewer supports $(S A=0.0940)$ than the second solution $(S A=594.2050)$, as can be seen in Fig. 13(a) and 13(b). Regarding the objective function $S Q$, there is no major change in the value of $S Q$, but there is a small improvement in the solution C, as it has a value of $S Q=0.0252$, while the solution A has a value of $S Q=0.0705$.

\subsubsection{BT vs $R A$}

For the $B T$ vs $R A$ problem, the selected representative solutions are indicated in Fig. 9 and Table 3 . All these solutions are non-dominated in terms of the two objectives.

Table 3 Representative non-dominated solutions for the $B T$ vs $R A$ problem

\begin{tabular}{crrrc}
\hline Solutions & \multicolumn{1}{c}{$\theta_{x}$} & \multicolumn{1}{c}{$\theta_{y}$} & \multicolumn{1}{c}{$B T$} & $R A$ \\
\hline K & 89.90 & 179.93 & 16.1397 & 39.2795 \\
J & 146.16 & 10.66 & 53.5297 & 19.7975 \\
B & 0.00 & 0.00 & 53.9148 & 17.0037 \\
F & 164.28 & 40.84 & 84.9517 & 16.8338 \\
D & 179.99 & 107.41 & 117.1316 & 14.9626
\end{tabular}




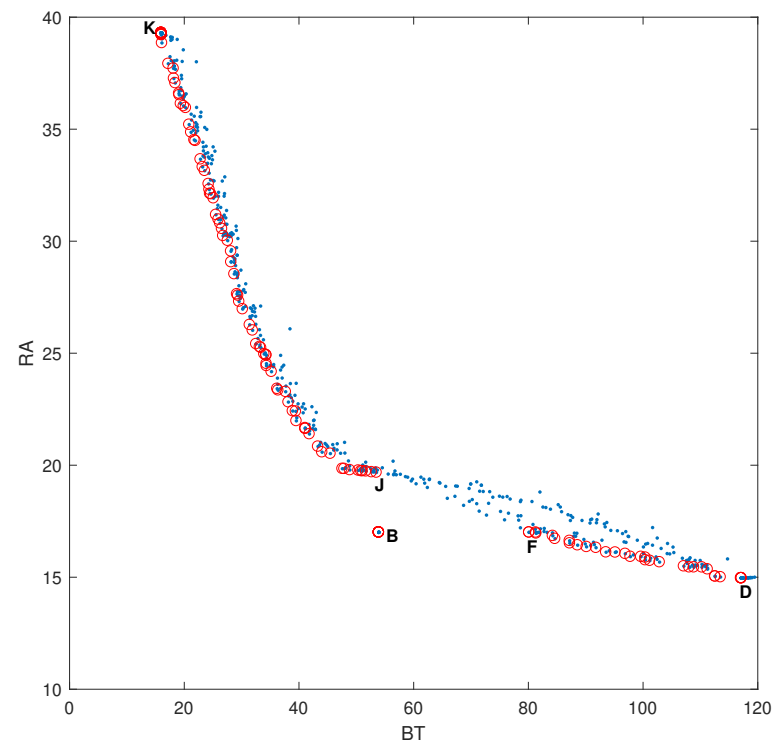

Fig. 9 Pareto front for the $B T$ vs $R A$ problem

In Fig. 9, the solutions $\mathrm{K}$ and $\mathrm{D}$ are the extremes of the Pareto front. There is an increase in the value of $B T$ from solution $\mathrm{K}$ to solution $\mathrm{D}$, and, on the other hand, from $\mathrm{K}$ to $\mathrm{D}$ a decrease in the value of $R A$ is observed. Thus, the objective functions $B T$ and $R A$ are, in this case, inversely proportional. Moreover, solution B represents a significant improve on $B T$ when compared with solution $\mathrm{F}$ at the expense of a very small degradation on the $R A$ value. So, solution $\mathrm{B}$ is an efficient solution close to the elbow of the Pareto front, representing a balanced trade-off. Figures 13(j) 13(i), 13(a) 13(e), and 13(c) allow to visualize the supports needed for printing the part.

\subsubsection{BT vs $S Q$}

Figure 10 shows the Pareto front for the $B T$ vs $S Q$ problem. The selected representative solutions are all non-dominated with exception of solution H. Table 4 presents the orientations angles and the objective values for the non-dominated solutions.

Solutions $\mathrm{K}$ and $\mathrm{C}$ are the extremes of the Pareto front. It can be observed, from solutions $\mathrm{K}$ to $\mathrm{C}$, an increase in the value of $B T$ and a small decrease in the value of $S Q$. Solutions $\mathrm{A}$ and $\mathrm{E}$ have the same value as $S Q$, although their orientations are similar, the values of $B T$ are different. Solution A requires less support structures providing less time $(B T)$ to print the part when compared to solution E, as shown in Fig. 13(a) and 13(d), Figures 13(a), 13(b), 13(c), 13(d), $13(\mathrm{~h})$ and $13(\mathrm{j})$ show a significant change in the orientation of the part. 


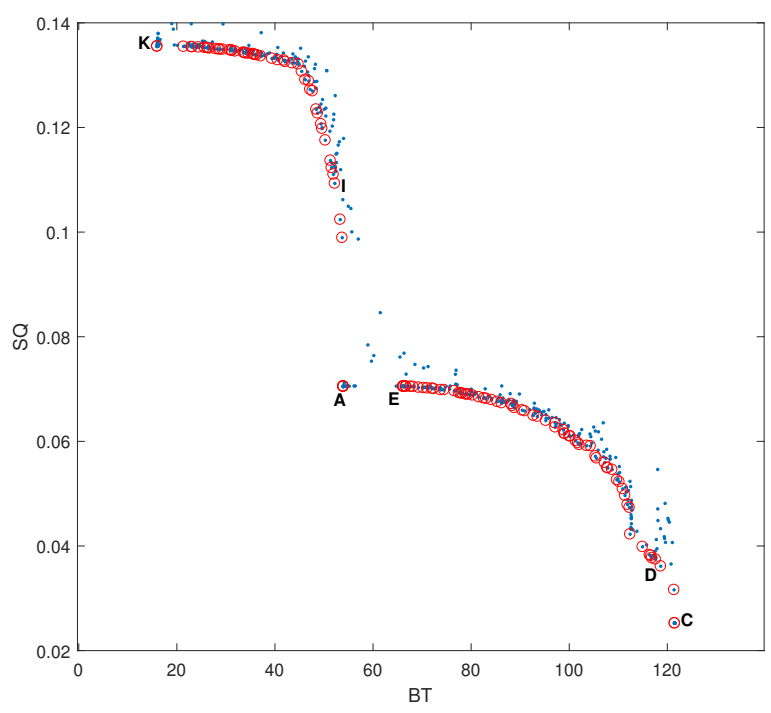

Fig. 10 Pareto front for the $B T$ vs $S Q$ problem

Table 4 Representative non-dominated solutions for the $B T$ vs $S Q$ problem

\begin{tabular}{crrrr}
\hline Solutions & \multicolumn{1}{c}{$\theta_{x}$} & \multicolumn{1}{c}{$\theta_{y}$} & \multicolumn{1}{c}{$B T$} & \multicolumn{1}{c}{$S Q$} \\
\hline $\mathrm{K}$ & 89.90 & 179.93 & 16.1397 & 0.1356 \\
$\mathrm{I}$ & 155.30 & 3.23 & 53.4056 & 0.1088 \\
$\mathrm{~A}$ & 0.00 & 0.02 & 53.9484 & 0.0705 \\
$\mathrm{E}$ & 0.00 & 7.58 & 66.3335 & 0.0705 \\
$\mathrm{D}$ & 179.99 & 107.41 & 117.1316 & 0.0370 \\
$\mathrm{C}$ & 94.80 & 90.00 & 121.5000 & 0.0252 \\
\hline
\end{tabular}

\subsubsection{RA vs $S Q$}

The Pareto front for $R A$ vs $S Q$ problem is represented in Fig. 11 pointing out the two non-dominated solutions $\mathrm{C}$ and D. From Table 5 it is clear that there is a decrease in the value of $S Q$ and a small increase in the value of $R A$, from solution D to solution C.

Table 5 Representative non-dominated solutions for the $R A$ vs $S Q$ problem

\begin{tabular}{crrrr}
\hline Solutions & \multicolumn{1}{c}{$\theta_{x}$} & \multicolumn{1}{c}{$\theta_{y}$} & $R A$ & $S Q$ \\
\hline $\mathrm{D}$ & 179.99 & 107.41 & 117.1316 & 0.0370 \\
$\mathrm{C}$ & 94.80 & 90.00 & 121.5000 & 0.0252
\end{tabular}

Figures 13(b) and 13(c) show a small change in the orientation of the part, with solution $\mathrm{C}$ needing fewer supports for printing, thus having a better surface quality $(S Q)$ compared to solution D. 


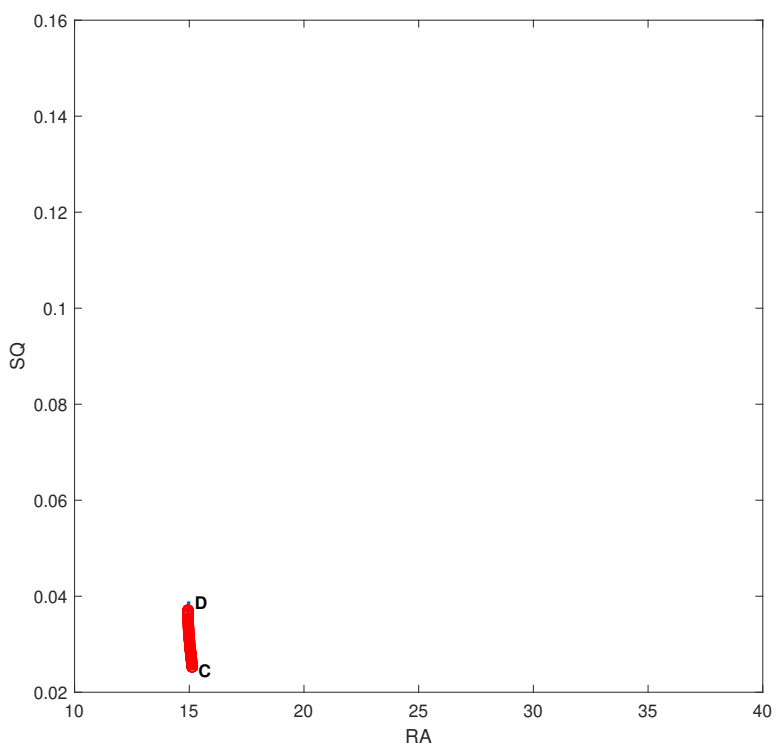

Fig. 11 Pareto front for the $R A$ vs $S Q$ problem

\subsection{Many-objective results}

The aim of the many-objective optimization problem is to, simultaneously, optimize the four objective functions $\left(S A, B T, R A\right.$ and $S Q$ ) considering $f_{1}=S A$, $f_{2}=B T, f_{3}=R A$ and $f_{4}=S Q$. The MATLAB ${ }^{\circledR}$ gamultiobj function was used to solve this many-objective problem with the same parameters used in the bi-objective problems, that is, population size is 50 and maximum number of generations is 600 . The problem was run for 30 times.

Table 6 presents all representative solutions selected for the bi-objective and many-objective problems. These solutions can be visualized in Fig. 13. All solutions presented in this table are non-dominated for the many-objective problem. Solution A has the lowest value of $S A$, conversely, solution L has the highest value. For solution $\mathrm{A}$, the value of $B T$ is greater than solution $\mathrm{L}$, since the latter is in a lying position, that is, the lower height of the part (lower $B T$ ), as can be seen in Fig. 13(a) and 13(k), Regarding the objective functions $R A$ and $S Q$, there are lower values of these in solution A, compared to the solution $\mathrm{L}$, since the fewer supports required $(S A)$, the less the surface roughness $(R A)$ and the higher the surface quality $(S Q)$.

The Pareto front for this many-objective problem is four-dimension surface that can not easily be represented in a single graph. Therefore, the graphs showing the 2D-projections of the Pareto front are presented in Fig. 12

In Fig. 12(a) 12(f) the representative solutions of Table 6 are shown in terms of the 2D-projections of the Pareto front. It is possible to observe that the nondominated solutions $\mathrm{C}, \mathrm{E}$ and $\mathrm{K}$ are dominated solutions in the bi-objective prob- 


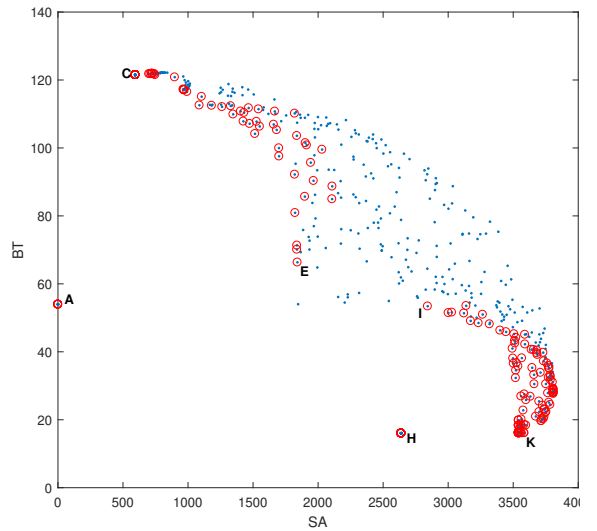

(a) Projected solutions on $S A$ and $B T$

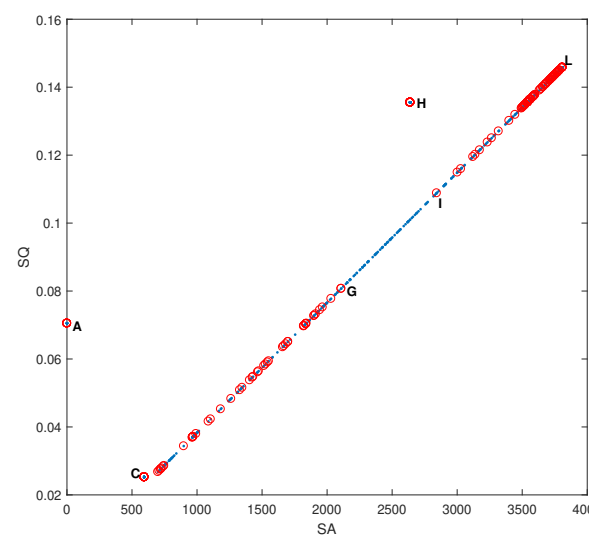

(c) Projected solutions on $S A$ and $S Q$

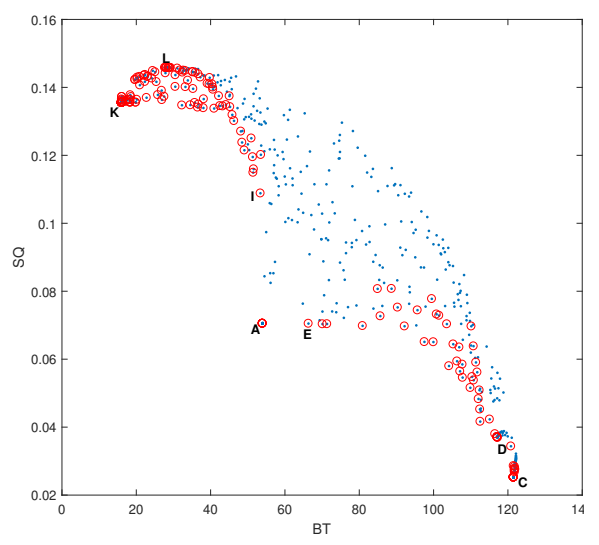

(e) Projected solutions on $B T$ and $S Q$

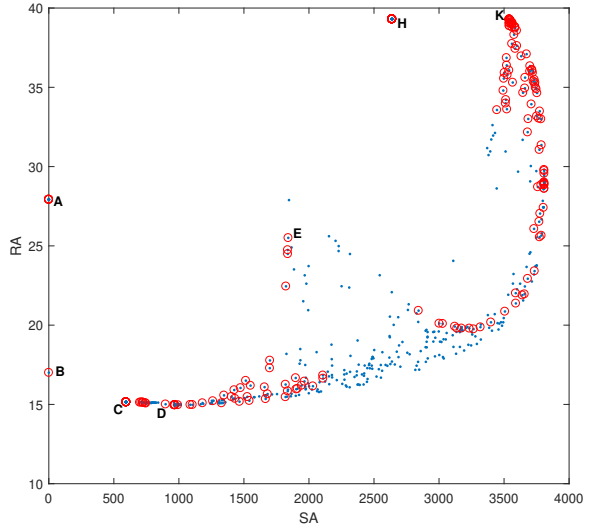

(b) Projected solutions on $S A$ and $R A$

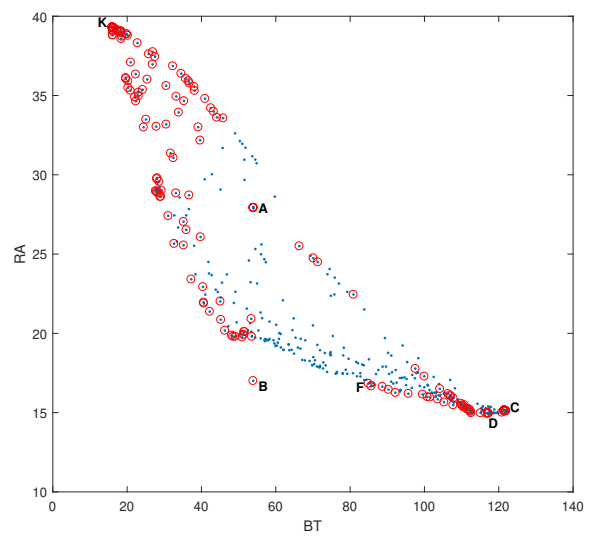

(d) Projected solutions on $B T$ and $R A$

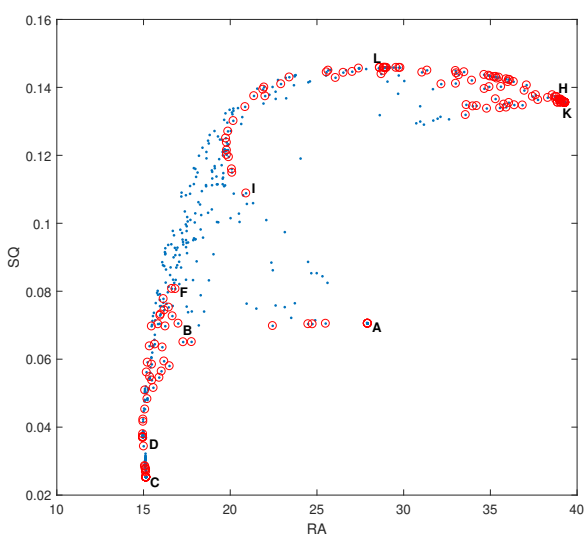

(f) Projected solutions on $R A$ and $S Q$

Fig. 12 Projected solutions of the many-objective problem 
lem $S A$ vs $B T$ (Fig. 7). Finally, solutions G and L were only found in the problem of optimizing the four objective functions simultaneously.

Table 6 Representative solutions for the many-objective problem

\begin{tabular}{crrrrrc}
\hline Solutions & \multicolumn{1}{c}{$\theta_{x}$} & \multicolumn{1}{c}{$\theta_{y}$} & \multicolumn{1}{c}{$S A$} & \multicolumn{1}{c}{$B T$} & $R A$ & \multicolumn{1}{c}{$S Q$} \\
\hline $\mathrm{A}$ & 0.00 & 0.02 & 0.0940 & 53.9484 & 27.9241 & 0.0705 \\
$\mathrm{~B}$ & 0.00 & 0.00 & 0.1313 & 53.9148 & 17.0037 & 0.0705 \\
$\mathrm{C}$ & 94.80 & 90.00 & 594.2050 & 121.5000 & 15.1459 & 0.0252 \\
$\mathrm{D}$ & 179.99 & 107.41 & 966.7508 & 117.1316 & 14.9626 & 0.0370 \\
$\mathrm{E}$ & 0.01 & 7.58 & 1841.1001 & 66.3335 & 25.5034 & 0.0705 \\
$\mathrm{~F}$ & 164.28 & 40.84 & 2107.7057 & 84.9517 & 16.8338 & 0.0807 \\
$\mathrm{G}$ & 162.15 & 43.15 & 2108.8774 & 88.6955 & 16.6365 & 0.0807 \\
$\mathrm{H}$ & 90.00 & 180.00 & 2637.4021 & 15.9888 & 39.3049 & 0.1355 \\
$\mathrm{I}$ & 155.30 & 3.23 & 2842.7707 & 53.4056 & 20.9137 & 0.1088 \\
$\mathrm{~J}$ & 146.16 & 10.66 & 3138.6630 & 53.5297 & 19.7975 & 0.1202 \\
$\mathrm{~K}$ & 89.90 & 179.93 & 3542.0142 & 16.1397 & 39.2795 & 0.1356 \\
$\mathrm{~L}$ & 68.20 & 179.91 & 3808.8381 & 27.6618 & 28.9808 & 0.1458 \\
\hline
\end{tabular}

\subsection{Discussion of the results}

The number of optimal solutions increases exponentially with a growing number of conflicting objectives. As expected, the solutions found for the single-objective problem were also found by the algorithm for the bi-objective and many-objective problems. These solutions are the extremes of the Pareto optimal fronts. Moreover, the Pareto optimal fronts of the bi-objective problems are the extremes of the 4Ddimensional Pareto front of the many-objective Pareto optimal front. So, some non-dominated solutions of the many-objective problem are dominated solutions of the bi-objective problems.

In the bi-objective optimization, some solutions were found repeatedly for different combinations of two objective functions. For the many-objective problem, additional optimal solutions were found that can not be attained using the biobjective optimization. This highlights the importance of considering all objectives simultaneously nevertheless the increasing complexity of the resulting multiobjective problem. Therefore, the number of Pareto optimal solutions increases and, consequently, the difficulty to choose between them also increases.

The ultimate goal is to provide to the decision-maker several optimal alternatives and information about the trade-offs. Visualization tools are crucial to give to the decision-maker more understanding and insights about the problem. Therefore, in order the complement the information given by the Pareto front, path value graphs were plotted to help the decision-maker to identify the relevant compromises and differentiate between the representative solutions chosen from the set of non-dominated solutions.

Figure 14 is the path value graph for the representative solutions in terms of the objectives. The horizontal lines represent the standardized objective values for the different trade-off solutions. As expected, all horizontal lines cross each other because they represent non-dominated solutions. Since all objectives are being minimized, the lowest standardized objective values correspond to the best 


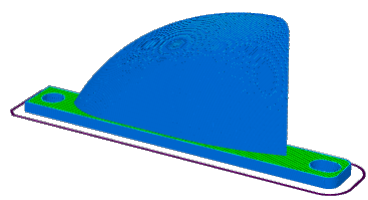

(a) A,B

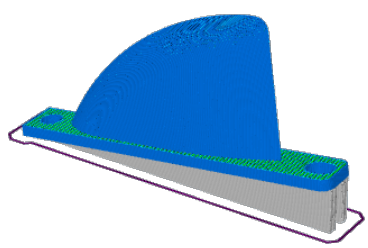

(d) E

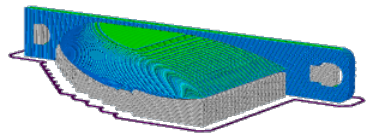

(g) $\mathrm{H}$

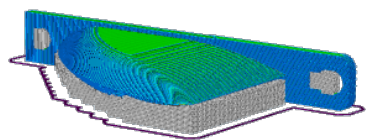

(j) $\mathrm{K}$

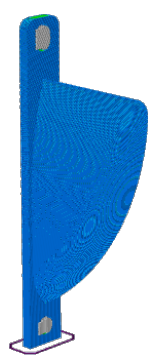

(b) $\mathrm{C}$

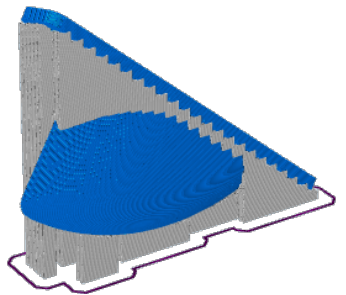

(e) $\mathrm{F}$

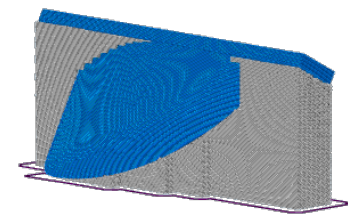

(h) I

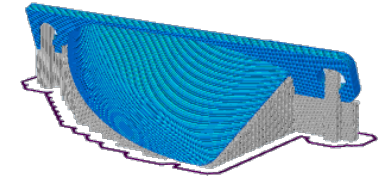

(k) L

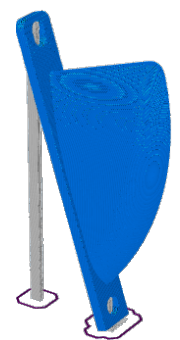

(c) $\mathrm{D}$

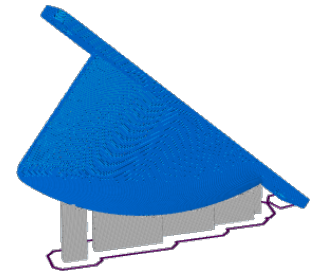

(f) $\mathrm{G}$

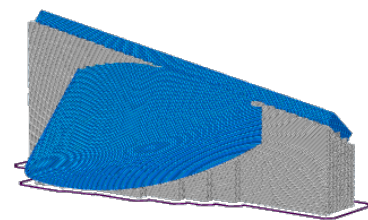

(i) $\mathrm{J}$

Fig. 13 Visualization of the representative solutions presented in Table 6

solutions. It can be observed that $\mathrm{B}$ is the best solution for $S A ; \mathrm{H}$ is the best solution in terms of $B T$; solution $\mathrm{D}$ has the best $R A$ value; and, $\mathrm{C}$ is the best solution for $S Q$. This graph highlights the trade-offs and also the similarity of solutions in terms of the objectives. For instance, solutions $\mathrm{C}$ and $\mathrm{D}$ are very similar. Solution $\mathrm{C}$ is better in terms of $S A$ and $S Q$, but slightly worse in terms of $B T$ and $R A$. Solution $\mathrm{E}$ is a balanced compromise between all objectives.

Figure 15 is the path value graph for objectives in terms of the representative solutions. The information in this graph complements the graph from Fig. 14. It can be observed that solution $\mathrm{H}$ has the best $B T$ value, but it is very bad in terms of $S A, R A$ and $S Q$. On the other hand, B is the best solution for SA and has reasonable values for the remaining objectives. Again, it is possible to observe that solution $\mathrm{E}$ has intermediate values of all objectives. 


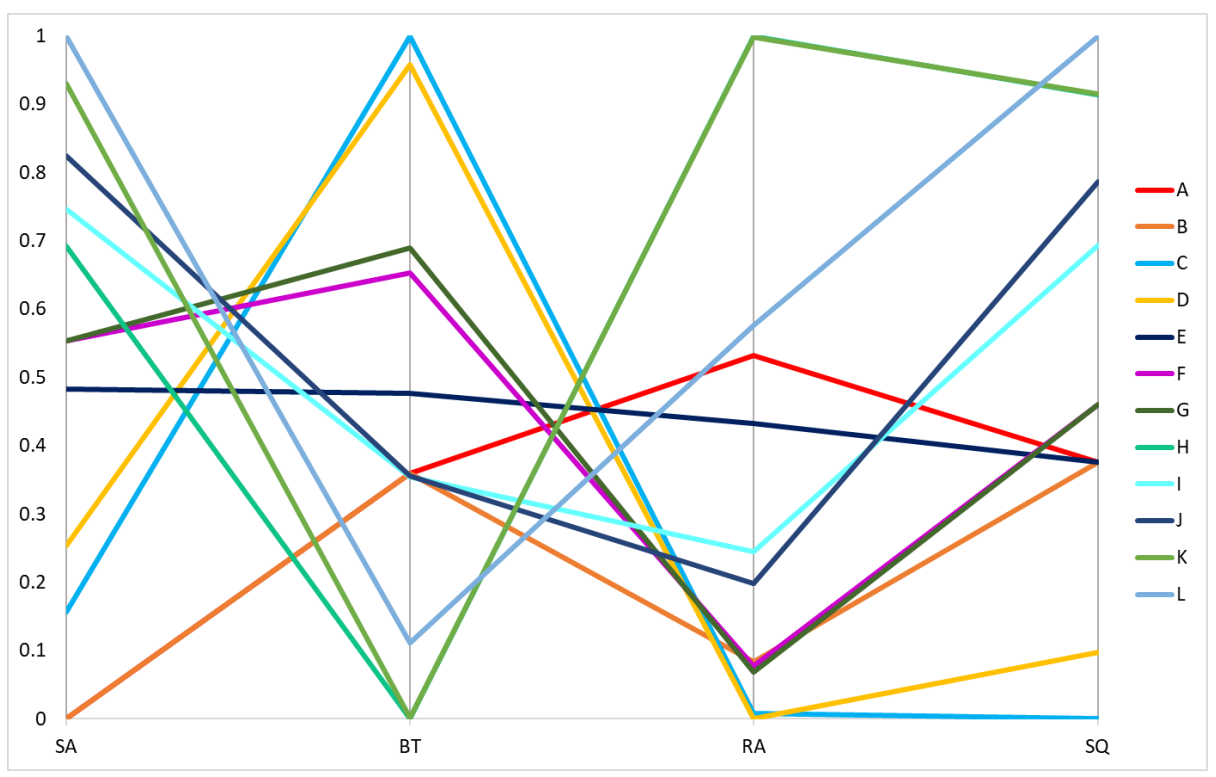

Fig. 14 Graph for all objective functions for the Fin model

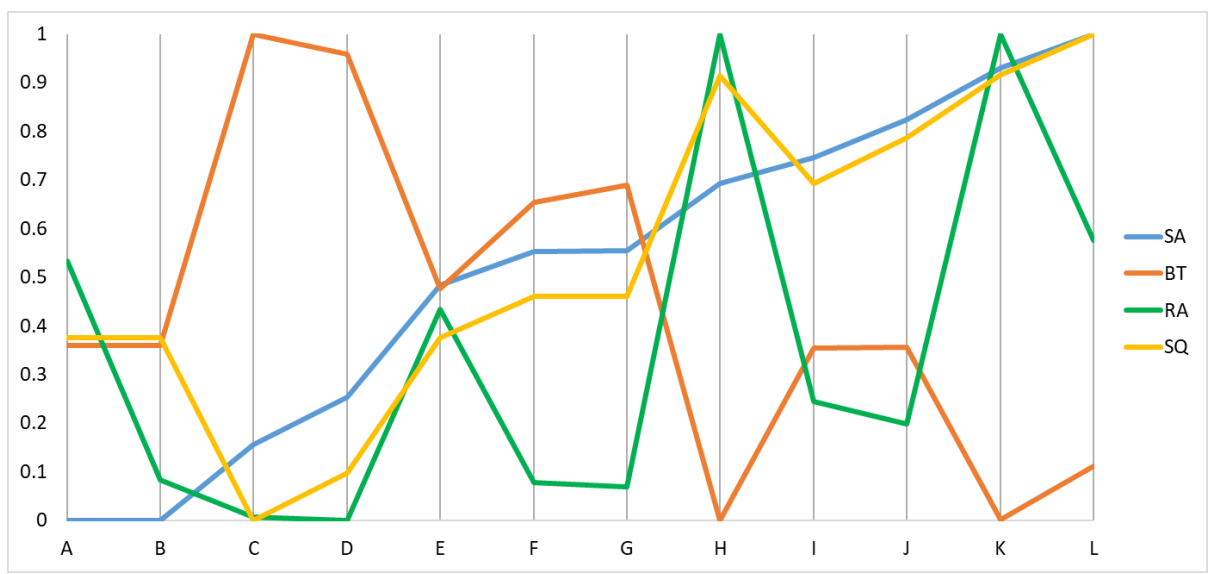

Fig. 15 Graph for all orientations for the Fin model

\section{Conclusions}

In this paper, a many-objective optimization approach for the build orientation problem of the Fin model is presented. The greatest contribution in this work is to assist the decision-maker in the $3 \mathrm{D}$ models orientation definition taking into account different factors. Four quality measures were considered: the total contact area of supports, build time, surface roughness and surface quality. Several optimization problems were formulated and solved using the NSGA-II algorithm implemented in the MATLAB ${ }^{\circledR}$ environment. 
First, a bi-objective problem with combinations of two objective functions was presented, $S A$ vs $B T, S A$ vs $R A, S A$ vs $S Q, B T$ vs $R A, B T$ vs $S Q$ and $R A$ vs $S Q$. Here, the Pareto fronts for each combination of functions were shown and the representative non-dominated solutions were identified. Then, a many-objective approach aiming to optimize the four measures simultaneously, $S A, B T, R A$ and $S Q$, was proposed. Additional optimal solutions were found that could not be attained using the bi-objective optimization.

The 2D-projections of the Pareto fronts of the many-objective problem were provided in order to select representative solutions. The results were analyzed using path value graphs in terms of the objectives and representative solutions to facilitate the identification of the compromises between the objectives and assist the decision-maker to choose a preferable build orientation.

The solutions showed the effectiveness of the proposed approach, since it was possible to find different optimal solutions considering the different criteria. This revealed a promising strategy to be taken into consideration to the future, when it is desired to print a $3 \mathrm{D}$ object in a short time, reduced material waste and with better surface finish. Finally, it was observed that, for all problems, the Pareto fronts have nonconvexities and discontinuities. This highlights the importance of formulating and solving this kind of problems as multi-objective problems.

In the future, multi-objective optimization using other objective functions and testing more difficult models will be performed.

Acknowledgements This work has been developed under the FIBR3D project - Hybrid processes based on additive manufacturing of composites with long or short fibers reinforced thermoplastic matrix (POCI-01-0145-FEDER-016414), supported by the Lisbon Regional Operational Programme 2020, under the PORTUGAL 2020 Partnership Agreement, through the European Regional Development Fund (ERDF). This work has been supported by FCT Fundação para a Ciência e Tecnologia within the R\&D Units Project Scope: UIDB/00319/2020.

\section{References}

Ahn, D.-K., Kwon, S.-M., \& Lee, S.-H. (2008). Expression for surface roughness distribution of fdm processed parts. In Smart manufacturing application, 2008. icsma 2008. international conference on (pp. 490-493).

Alexander, P., Allen, S., \& Dutta, D. (1998). Part orientation and build cost determination in layered manufacturing. Computer-Aided Design, 30(5), 343356 .

Asadi-Eydivand, M., Solati-Hashjin, M., Fathi, A., Padashi, M., \& Osman, N. A. A. (2016). Optimal design of a 3d-printed scaffold using intelligent evolutionary algorithms. Applied Soft Computing, 39, 36-47.

Behnam, N. (2011). Surface roughness estimation for fdm systems (Unpublished doctoral dissertation). MSc thesis, Ryerson University, Toronto.

Brika, S. E., Zhao, Y. F., Brochu, M., \& Mezzetta, J. (2017). Multi-objective build orientation optimization for powder bed fusion by laser. Journal of Manufacturing Science and Engineering, 139(11), 111011.

Byun, H. S., \& Lee, K. H. (2006). Determination of optimal build direction in rapid prototyping with variable slicing. The International Journal of Advanced Manufacturing Technology, 28(3-4), 307. 
Campbell, R. I., Martorelli, M., \& Lee, H. S. (2002). Surface roughness visualisation for rapid prototyping models. Computer-Aided Design, 34(10), 717-725.

Canellidis, V., Dedoussis, V., Mantzouratos, N., \& Sofianopoulou, S. (2006). Preprocessing methodology for optimizing stereolithography apparatus build performance. Computers in industry, 57(5), 424-436.

Canellidis, V., Giannatsis, J., \& Dedoussis, V. (2009). Genetic-algorithm-based multi-objective optimization of the build orientation in stereolithography. The International Journal of Advanced Manufacturing Technology, 45(7-8), 714-730.

Cheng, W., Fuh, J., Nee, A., Wong, Y., Loh, H., \& Miyazawa, T. (1995). Multi-objective optimization of part-building orientation in stereolithography. Rapid Prototyping Journal, 1(4), 12-23.

Chong, L., Ramakrishna, S., \& Singh, S. (2018). A review of digital manufacturingbased hybrid additive manufacturing processes. The International Journal of Advanced Manufacturing Technology, 95(5-8), 2281-2300.

Das, P., Chandran, R., Samant, R., \& Anand, S. (2015). Optimum part build orientation in additive manufacturing for minimizing part errors and support structures. Procedia Manufacturing, 1, 343-354.

Deb, K. (2001). Multi-objective optimization using evolutionary algorithms. New York, NY, USA: John Wiley \& Sons, Inc.

Frank, D., \& Fadel, G. (1995). Expert system-based selection of the preferred direction of build for rapid prototyping processes. Journal of Intelligent Manufacturing, 6(5), 339-345.

Ga, B., Gardan, N., \& Wahu, G. (2019). Methodology for part building orientation in additive manufacturing. Computer-Aided Design $\&$ Applications, 16(1), 113128.

Goldberg, D. E. (1989). Genetic algorithms in search optimization, and machine learning. New York: Addison Wesley Publishing Co. Inc.

Golmohammadi, A., \& Khodaygan, S. (2019). A framework for multi-objective optimisation of $3 \mathrm{~d}$ part-build orientation with a desired angular resolution in additive manufacturing processes. Virtual and Physical Prototyping, 14(1), 19-36.

Gurrala, P. K., \& Regalla, S. P. (2014). Multi-objective optimisation of strength and volumetric shrinkage of fdm parts: a multi-objective optimization scheme is used to optimize the strength and volumetric shrinkage of fdm parts considering different process parameters. Virtual and Physical Prototyping, 9(2), 127-138.

Jaiswal, P., Patel, J., \& Rai, R. (2018). Build orientation optimization for additive manufacturing of functionally graded material objects. The International Journal of Advanced Manufacturing Technology, 1-13.

Jibin, Z. (2005). Determination of optimal build orientation based on satisfactory degree theory for rpt. In Computer aided design and computer graphics, 2005. ninth international conference on (pp. 6-pp).

Kattethota, G., \& Henderson, M. (1998). A visual tool to improve layered manufacturing part quality. In Proceedings of solid freeform fabrication symposium (pp. 327-334).

Khan, I., Mateus, A., Lorger, C. S. K., \& Mitchell, G. R. (2017). Part specific applications of additive manufacturing. Procedia Manufacturing, 12, 89-95. 
Li, A., Zhang, Z., Wang, D., \& Yang, J. (2010). Optimization method to fabrication orientation of parts in fused deposition modeling rapid prototyping. In 2010 international conference on mechanic automation and control engineering (pp. 416-419).

Livesu, M., Ellero, S., Martínez, J., Lefebvre, S., \& Attene, M. (2017). From $3 \mathrm{~d}$ models to $3 \mathrm{~d}$ prints: an overview of the processing pipeline. Computer Graphics Forum, 36(2), 537-564.

Mason, A. (2007). Multi-axis hybrid rapid prototyping using fusion deposition modeling. ProQuest.

Masood, S. H., Rattanawong, W., \& Iovenitti, P. (2000). Part build orientations based on volumetric error in fused deposition modelling. The International Journal of Advanced Manufacturing Technology, 16(3), 162-168.

MATLAB. (2019). version 9.6.0.1214997 (r2019a). Natick, Massachusetts: The MathWorks Inc.

Matos, M. A., Rocha, A. M. A., Costa, L. A., \& Pereira, A. I. (2019). A multiobjective approach to solve the build orientation problem in additive manufacturing. In International conference on computational science and its applications (pp. 261-276).

Matos, M. A., Rocha, A. M. A., \& Pereira, A. I. (2020). Improving additive manufacturing performance by build orientation optimization. The International Journal of Advanced Manufacturing Technology, 1-13.

Matos, M. A., Rocha, A. M. A. C., \& Pereira, A. I. (2019). On optimizing the build orientation problem using genetic algorithm. In Aip conference proceedings (Vol. 2116, p. 220006).

Mirzendehdel, A. M., \& Suresh, K. (2016). Support structure constrained topology optimization for additive manufacturing. Computer-Aided Design, 81, 1-13.

Mohan Pandey, P., Venkata Reddy, N., \& Dhande, S. G. (2003). Slicing procedures in layered manufacturing: a review. Rapid prototyping journal, 9(5), 274-288.

Pandey, P. M. (2010). Rapid prototyping technologies, applications and part deposition planning. Retrieved October, 15, 550-555.

Pandey, P. M., Reddy, N. V., \& Dhande, S. G. (2003). Improvement of surface finish by staircase machining in fused deposition modeling. Journal of materials processing technology, 132(1-3), 323-331.

Pereira, S., Vaz, A., \& Vicente, L. (2018). On the optimal object orientation in additive manufacturing. The International Journal of Advanced Manufacturing Technology, 98(5-8), 1685-1694.

Phatak, A. M., \& Pande, S. (2012). Optimum part orientation in rapid prototyping using genetic algorithm. Journal of manufacturing systems, 31(4), 395-402.

Ransikarbum, K., Ha, S., Ma, J., \& Kim, N. (2017). Multi-objective optimization analysis for part-to-printer assignment in a network of $3 \mathrm{~d}$ fused deposition modeling. Journal of Manufacturing Systems, 43, 35-46.

Rocha, A. M. A. C., Pereira, A. I., \& Vaz, A. I. F. (2018). Build orientation optimization problem in additive manufacturing. In International conference on computational science and its applications (pp. 669-682).

SIMPLIFY3D, I. S. S. (2017). version 4.0.0 (2017). Legal Dept, Simplify3D: Simplify3D LLC.

Sumalatha, M., \& Rao, V. (2018). Experimental evaluation of the best orientation of taper plug to minimize support material required in $3 \mathrm{~d}$ printing ( $\mathrm{fdm}$ ). Journal of Innovation in Mechanical Engineering, 1(2), 11-15. 
Taufik, M., \& Jain, P. K. (2013). Role of build orientation in layered manufacturing: a review. International Journal of Manufacturing Technology and Management, 27(1-3), 47-73.

Thrimurthulu, K., Pandey, P. M., \& Reddy, N. V. (2004). Optimum part deposition orientation in fused deposition modeling. International Journal of Machine Tools and Manufacture, 44(6), 585-594.

Vahabli, E., \& Rahmati, S. (2017). Hybrid estimation of surface roughness distribution in fdm parts using analytical modeling and empirical investigation. The International Journal of Advanced Manufacturing Technology, 88(58), 2287-2303.

Wang, W. M., Zanni, C., \& Kobbelt, L. (2016). Improved surface quality in $3 \mathrm{~d}$ printing by optimizing the printing direction. Computer graphics forum, $35(2), 59-70$.

Xu, F., Wong, Y., Loh, H., Fuh, J., \& Miyazawa, T. (1997). Optimal orientation with variable slicing in stereolithography. Rapid Prototyping Journal, 3(3), $76-88$.

Zhang, X., Le, X., Panotopoulou, A., Whiting, E., \& Wang, C. C. (2015). Perceptual models of preference in $3 \mathrm{~d}$ printing direction. ACM Transactions on Graphics (TOG), 34(6), 215.

Zhang, Y., \& Bernard, A. (2013). Using am feature and multi-attribute decision making to orientate part in additive manufacturing. In High value manufacturing: Advanced research in virtual and rapid prototyping. proceedings of the 6th international conference on advanced research in virtual and rapid prototyping (pp. 411-416).

Zhang, Y., Bernard, A., Gupta, R. K., \& Harik, R. (2016). Feature based building orientation optimization for additive manufacturing. Rapid Prototyping Journal.

Zhang, Y., Bernard, A., Harik, R., \& Karunakaran, K. (2017). Build orientation optimization for multi-part production in additive manufacturing. Journal of Intelligent Manufacturing, 28(6), 1393-1407.

Zhang, Y., De Backer, W., Harik, R., \& Bernard, A. (2016). Build orientation determination for multi-material deposition additive manufacturing with continuous fibers. Procedia Cirp, 50(2016), 414-419. 